\title{
Evaluation of rapid techniques for the detection of mycobacteria in sputum with scanty bacilli or clinically evident, smear negative cases of pulmonary and extra-pulmonary tuberculosis
}

\author{
Pottathil Shinu ${ }^{1 /+}$, Anroop Nair ${ }^{2}$, Varsha Singh ${ }^{1}$, Surinder Kumar', Rajesh Bareja ${ }^{1}$ \\ ${ }^{1}$ Department of Microbiology, M.M. Institute of Medical Sciences and Research \\ ${ }^{2}$ M.M. College of Pharmacy, M.M. University, Mullana, Ambala, India - 134007
}

The objective of the current study was to compare two rapid methods, the BBL Mycobacteria Growth Indicator Tube $\left(M_{G I T^{T M}}\right)$ and Biotec FASTPlaque TB ${ }^{\mathrm{TM}}$ (FPTB) assays, with the conventional Löwenstein-Jensen (LJ) media assay to diagnose mycobacterial infections from paucibacillary clinical specimens. For evaluation of the clinical utility of the $B B L M G I T^{T M}$ and FPTB assays, respiratory tract specimens $(n=208)$, with scanty bacilli or clinically evident, smear negative cases and non-respiratory tract specimens $(n=119)$ were analyzed and the performance of each assay was compared with LJ media. MGIT and FPTB demonstrated a greater sensitivity (95.92\% and 87.68\%), specificity (94.59\% and $98.78 \%$ ), positive predictive value (94.91\% and $99.16 \%)$ and negative predictive value $(96.56 \%$ and $90.92 \%)$, respectively, compared to LJ culture for both respiratory tract and non-respiratory tract specimens. However, the FPTB assay was unable to detect nontuberculous mycobacteria and few Mycobacterium tuberculosis complex cases from paucibacillary clinical specimens. It is likely that the analytical sensitivity of FPTB is moderately low and may not be useful for the direct detection of tuberculosis in paucibacillary specimens. The current study concluded that MGIT was a dependable, highly efficient system for recovery of $\mathrm{M}$. tuberculosis complexes and nontuberculous mycobacteria from both respiratory and non-respiratory tract specimens in combination with LJ media.

Key words: Biotec FASTPlaque TB ${ }^{\mathrm{TM}}$ assay - BBL MGIT ${ }^{\mathrm{TM}}$ - non-respiratory - respiratory tract specimens

The recent report by the World Health Organization (WHO 2010) reveals that one third of the world's population is currently infected with tuberculosis (TB) and the number of incidents is alarming in Southeast Asia, which accounts for approximately $35 \%$ of global cases. The diagnosis of TB and other mycobacterial infections in the early stage of the disease is of utmost importance to decrease the incidence. Therefore, it is necessary for clinical microbiology laboratories to detect and identify mycobacteria from human clinical material rapidly. Microscopy and culture are generally considered to be the authoritative methods for the laboratory diagnosis of TB. However, smear microscopy is limited due to low sensitivity in paucibacillary specimens (Marei et al. 2003). On the other hand, culture remains the gold standard for diagnosis of mycobacterial infections, although it is time consuming and prone to contamination (McNerney 1996, Nancy et al. 2000). Various culture techniques have been investigated during the last few decades and, at present, several research groups are exploring the possibility to improve their efficiency (Dorman 2010, Kandi \& Madhavi 2010). Several manual and automated systems have been introduced specifically to reduce the time of detection and identification of mycobacteria in clinical specimens. The Mycobacteria Growth Indicator Tube (MGIT)

+Corresponding author: shinup1983@gmail.com

Received 31 January 2011

Accepted 2 June 2011
(Becton Dickinson) provides rapid recovery of mycobacteria from clinical specimens (Badak et al. 1996). Alternatively, the rapid detection and counting of Mycobacterium tuberculosis complexes from both respiratory and non-respiratory tract specimens may be achieved using the Biotec FASTPlaque TB ${ }^{\mathrm{TM}}$ (FPTB) assay (Biotec Laboratories Ltd, UK), which utilizes a phage amplification technology (Albert et al. 2002, Marei et al. 2003).

Literature indicates that most of the studies on the diagnosis of TB and recovery of mycobacteria have been carried out in pulmonary and extra-pulmonary specimens (Hanna et al. 1999, Hillemann et al. 2006). Several nucleic acid amplification and nucleic acid hybridization techniques have been introduced, but they were found to be less sensitive than culture-based techniques and are often complicated due to the presence of inhibitors (Nancy et al. 2000, Cheng et al. 2005). Alternatively, common techniques, such as the acid-fast bacillus (AFB) smear and Löwenstein-Jensen (LJ) cultures, have limitations, such as low sensitivity, and require prolonged incubation (typically weeks). However, rapid techniques, like MGIT, are widely used in the diagnosis of TB from both pulmonary and extra-pulmonary specimens. The objective of the current study was to compare two rapid methods, the BBL MGIT ${ }^{\text {TM }}$ and FPTB assays, with the conventional LJ media culture assay to diagnose mycobacterial infections from clinical specimens with scanty bacilli. Moreover, to our knowledge, this is the first report that investigated the performance of the BBL MGIT $^{\mathrm{TM}}$ and FPTB assays compared to conventional LJ media for use in the recovery of mycobacteria from pulmonary (sputum with scanty bacilli or clinically evident, smear negative cases) and extra-pulmonary TB. 
This study was approved by the Maharishi Markendeshwar University Ethical Committee. The study received a waiver of informed consent because the samples used were collected for use in approved routine tests on LJ media and the BBL MGIT ${ }^{\mathrm{TM}}$. Tests on FPTB were performed in parallel with the approved routine culture on LJ media and MGIT. The study was carried out from March 2008-July 2010 at Maharishi Markendeshwar Institute of Medical Sciences and Research, Mullana, Ambala, India, a 750-bed tertiary care hospital that also operates as a centre for the Revised National Tuberculosis Control Program. All clinically suspected fresh cases of extra-pulmonary and pulmonary TB, sputum with scanty bacilli (1-9 bacilli/100 fields) or clinically evident, smear negative cases, were included in the study. Patients receiving or who received anti-TB treatment in the previous month and sputum samples suggestive of saliva were excluded from this study. A total of 327 samples were examined and were found to be composed of sputum, bronchoalveolar lavage, cerebrospinal fluid, fine needle aspiration cytology specimens, pus, tissue biopsy, pleural fluid, pericardial fluid and ascetic fluid.

All clinical specimens in a liquid state were digested and decontaminated by the N-acetyl-L-cysteine- $\mathrm{NaOH}$ method with a final $\mathrm{NaOH}$ concentration of $1 \%$ (Kent \& Kubica 1985). After centrifugation, AFB smears were made for all clinical specimens studied using the sediment and stained with the Ziehl-Neelsen acid fast method; grading of smears was carried out as described by Kent and Kubica (1985). The remaining sediment was suspended in 1-2 $\mathrm{mL}$ of sterile $0.67 \mathrm{M}$ phosphate buffer $(\mathrm{pH} \mathrm{6.8)} \mathrm{and}$ vortexed for $15 \mathrm{~s}$. This suspension was used for culture media inoculation. All liquid media were inoculated first, followed by inoculation of egg-based solid media, such as LJ media. Three types of culture media were inoculated.

The BBL MGIT ${ }^{\mathrm{TM}}$ culture tubes were inoculated with $0.5 \mathrm{~mL}$ of the processed specimens using a needle and syringe and incubated at $37^{\circ} \mathrm{C}$, according to the instruction from the manufacturer. Readings were taken daily for the first three weeks and once a week thereafter for culture positivity until the end of six weeks using the Manual BBL MGIT ${ }^{\mathrm{TM}}$ system. The tubes showing positive growth were further subjected to AFB smear and subculture on LJ media. Confirmation of $M$. tuberculosis complexes was made by para-nitro-benzoic acid (PNB) testing (Allen 1984). The presence of M. tuberculosis complexes was established by lack of growth on PNB containing media.

The manufacturer's instructions were strictly followed to process respiratory tract specimens for the FASTPlaque $\mathrm{TB}^{\mathrm{TM}}$ kit, but minor alterations were made when processing non-respiratory tract specimens because this kit was not recommended for non-respiratory specimens. Briefly, both a positive (serial dilution of $1 \mathrm{~mL}$ of $10^{-2}$, $10^{-4}, 10^{-6}$ Mycobacterium smegmatis) and negative $(1 \mathrm{~mL}$ of FPTB medium broth) control were prepared. FPTB medium $(1 \mathrm{~mL})$ was mixed with decontaminated and concentrated sediment $(1 \mathrm{~mL})$ in a reaction vessel and the suspension was incubated overnight at $37^{\circ} \mathrm{C}$. However, for non-respiratory specimens, the incubation time was prolonged to $48 \mathrm{~h}$ to enhance enrichment. An Actiphage solution $(100 \mathrm{~mL})$ containing mycobacteriophages was added and incubated for $1 \mathrm{~h}$ to allow the infection to take place. Then, a virucide solution (Virusol, $100 \mathrm{~mL}$ ) was added and incubated at room temperature for $15 \mathrm{~min}$. The excess of virucide solution was neutralized by adding $5 \mathrm{~mL}$ of FPTB medium followed by addition of helper cells $(1 \mathrm{~mL})$. This suspension was mixed well and added to a Petri dish overlayed with $5 \mathrm{~mL}$ of molten agar. Plates were rotated several times to mix the solution and allowed to set, followed by overnight incubation (approximately $15-18 \mathrm{~h}$ ) at $37^{\circ} \mathrm{C}$. The results were interpreted according to the manufacturer's instructions.

The processed specimen $(0.1 \mathrm{~mL})$ was inoculated on two slants of LJ egg medium, with one containing glycerol and the other containing sodium pyruvate. These slants were incubated at $37^{\circ} \mathrm{C}$ for a maximum of eight weeks and read once weekly. An individual medium was considered positive upon appearance of colonies on the surface and confirmed by positive AFB smear. Further confirmation of $M$. tuberculosis complex was made by PNB testing (Allen 1984). Reference strains, such as M. tuberculosis ATCC 27294, Mycobacterium kansasii ATCC 12478, Pseudomonas aeruginosa ATCC 27853 and Staphylococcus epidermidis ATCC 12228 were used for quality control on each new lot of MGIT. The sensitivity, specificity, positive predictive value (PPV) and negative predictive value (NPV) for the AFB smear, phage assay and BBL MGIT ${ }^{\mathrm{TM}}$ were calculated and compared with LJ culture. The following formula were used for calculations. Sensitivity was true positives/(true positives + false negatives) x 100, specificity was true negatives/(true negatives + false positives) $\mathrm{x} 100$, PPV was true positives/ (true positives + false positives) $\times 100$ and NPV was true negatives/(true negatives + false negatives) x 100 .

In the current study, a total of 327 specimens were processed for microscopy (AFB smear) and culture (MGIT, FPTB and LJ media). The distributions of all the specimens processed by FPTB, BBL MGIT ${ }^{\mathrm{TM}}$ and LJ culture techniques are summarized in Table I. The comparison of LJ media with AFB smear, FPTB and BBL MGIT ${ }^{\mathrm{TM}}$ in both respiratory and non-respiratory tract specimens is summarized in Table II. Out of 327 specimens processed, a total of 189 (respiratory tract, $\mathrm{n}=145$, non-respiratory tract, $\mathrm{n}=44$ ) isolates were obtained by all three culture methods (FPTB assay, BBL MGIT $^{\mathrm{TM}}$ and LJ). The results of the validation (average value) using the AFB smear method for the FPTB and BBL MGIT ${ }^{\mathrm{TM}}$ assays indicate moderate sensitivity (65.99\%), greater specificity $(100 \%)$, PPV $(100 \%)$ and NPV $(80.52 \%)$ with respect to LJ media for both respiratory tract and non-respiratory tract specimens. The validation data observed in the AFB smear in non-respiratory tract specimens indicated comparatively low sensitivity (Table II), which is likely due to the low content of bacilli in the specimens.

Data observed using the MGIT technique are presented in Table II. It is apparent that the MGIT technique provided better recovery of both $M$. tuberculosis complexes and nontuberculous mycobacteria from all specimens processed [respiratory (63.46\%) and non-respiratory (35.29\%)], compared to the other methods (LJ media and FPTB). This 
higher recovery rate could be due to the inoculum size, quality of media and the overall system; these results agree with those in previous studies (Hanna et al. 1999, Hillemann et al. 2006). Despite the advantages of broth-based cultivation systems, conventional culture media still play an important role in the recovery of mycobacteria from clinical specimens and are recommended for use along with a liquid medium (Kent \& Kubica 1985).

\section{TABLE I}

Distribution of all samples processed by Biotec FASTPlaque TB ${ }^{\mathrm{TM}}$ assay, BBL Mycobacteria Growth Indicator Tube and conventional culture technique $(\mathrm{n}=327)$

\begin{tabular}{lc}
\hline Specimen & (n) \\
\hline Respiratory tract specimens & 201 \\
Sputum & 7 \\
Bronchoalveolar lavage & \\
Non-respiratory tract specimens & 8 \\
Cerebrospinal fluid & 41 \\
Fine needle aspiration cytology & 26 \\
Pus & 20 \\
Tissue biopsy & 19 \\
Pleural fluid & 1 \\
Pericardial fluid & 4 \\
Ascetic fluid & 327 \\
\hline Total & \\
\hline
\end{tabular}

In general, it is accepted that liquid media are more susceptible to contamination than solid media (Hanna et al. 1999, Hillemann et al. 2006). However, in the current study, the number of contaminated cultures (MGIT) was found to be surprisingly low, probably due to the addition of the MGIT PANTA mixture and $\mathrm{NaOH}$ (final concentration $1 \%$ ). Reports indicated that the overall time required for the detection of $M$. tuberculosis complexes (for smear positive cases) was five-20 days and 10-45 days in the MGIT and LJ techniques, respectively (Pfyffer et al. 1997). This statement was substantiated in this study, except in two cases in which there were late positive cultures (52 days) on LJ media. In the case of smear negative specimens, the time to detect the $M$. tuberculosis complex when using the MGIT and LJ assays were found to be six46 days and 17-47 days, respectively. This prolonged incubation period is probably due to the paucibacillary nature of the specimen, as described by Pfyffer et al. (1997). Interestingly, the time of detection in liquid media exceeded that observed in solid media for nontuberculous mycobacteria (20-50 days for MGIT and 20-40 days for LJ media). All the AFB-positive non-respiratory tract specimens $(\mathrm{n}=16)$ showed growth in MGIT. Further, a higher number of validated results were observed in MGIT (sensitivity $95.92 \%$, specificity $94.59 \%$, PPV $94.91 \%$ and NPV $96.56 \%$ ) with respect to LJ culture for both respiratory tract and non-respiratory tract specimens.

Most current methods of diagnosis are either intricate or expensive. However, the phage assay is a simple, rapid technique that does not require any expensive instrumentation and can be employed for routine analysis in mycobacteriology laboratories (Albert et al. 2002). Moreover, this assay is relatively safe, as a greater amount of

TABLE II

Comparison of acid-fast bacillus (AFB) smear, Biotec FASTPlaque TB ${ }^{\text {TM }}$ assay (FPTB) and BBL Mycobacteria Growth Indicator Tube (MGIT) with Löwenstein-Jensen (LJ) culture in respiratory and non-respiratory tract specimens

\begin{tabular}{|c|c|c|c|c|c|c|c|c|}
\hline \multirow[b]{2}{*}{ Type of specimen } & \multirow[b]{2}{*}{ Methods } & \multicolumn{3}{|c|}{$\begin{array}{l}\text { Specimens } \\
\text { (n) }\end{array}$} & \multirow{2}{*}{$\begin{array}{c}\text { Sensitivity } \\
(\%)\end{array}$} & \multirow{2}{*}{$\begin{array}{c}\text { Specificity } \\
(\%)\end{array}$} & \multirow{2}{*}{$\begin{array}{l}\text { PPV } \\
(\%)\end{array}$} & \multirow{2}{*}{$\begin{array}{l}\text { NPV } \\
(\%)\end{array}$} \\
\hline & & & Positive & Negative & & & & \\
\hline \multirow[t]{6}{*}{ Respiratory tract ${ }^{a}$} & AFB & True & 109 & 81 & 85.83 & 100 & 100 & 81.82 \\
\hline & & False & - & 18 & - & - & - & - \\
\hline & MGIT & True & 132 & 70 & 98.51 & 94.59 & 98.51 & 97.22 \\
\hline & & False & 4 & 2 & - & - & - & - \\
\hline & FPTB & True & 117 & 80 & 92.86 & 97.56 & 98.32 & 89.89 \\
\hline & & False & 2 & 9 & - & - & - & - \\
\hline \multirow[t]{6}{*}{ Non-respiratory $\operatorname{tract}^{b}$} & AFB & True & 18 & 80 & 46.15 & 100 & 100 & 79.21 \\
\hline & & False & - & 21 & - & - & - & \\
\hline & MGIT & True & 42 & 70 & 93.33 & 94.59 & 91.30 & 95.89 \\
\hline & & False & 4 & 3 & - & - & - & - \\
\hline & FPTB & True & 32 & 80 & 82.05 & 100 & 100 & 91.95 \\
\hline & & False & - & 7 & - & - & - & - \\
\hline
\end{tabular}

$a$ : number of specimens positive (127) and negative (81) in LJ culture; $b$ : number of specimens positive (39) and negative (80) in LJ culture; NPV: negative predictive value; PPV: positive predictive value. 
bacilli develop into non-infective mycobacteriophages. In contrast, culture techniques release a greater number of infective agents during the assay procedure (Wilson et al. 1997). The limitations of the phage assay include an inability to distinguish $M$. tuberculosis complexes, a requirement for a confirmatory test to identify the species and reduced efficiency when sputum contains $>$ $10 \%$ blood or if the patient is on anti-tubercular treatment (Otive-Igbuzor 2002). In the current study, the results of microscopy were available in a short time $(1.5 \mathrm{~h})$, while in the case of LJ culture, the required period of detection was found to be four-eight weeks. However, for the phage assay, clear zones known as plaques (Figure) were accessible in less than 48-72 $\mathrm{h}$. The number of plaques formed in cases of positive specimens was found to be $\sim 35-300$ and was highly correlated with the positivity rate of the specimen. Moreover, the number of plaques is directly proportional to viable bacilli in the clinical specimen (Biotec Laboratories 2000a).

Of the $127 \mathrm{LJ}$ positive respiratory tract specimen cultures, results concurred with 115 FPTB positive cases. Furthermore, in all non-respiratory specimens processed $(\mathrm{n}=119)$, a greater number $(\mathrm{n}=44)$ of mycobacteria isolates were obtained. Among these, 42, 39 and 32 isolates were detected by MGIT, LJ and FPTB, respectively. Indeed, a higher sensitivity $(87.68 \%)$, specificity $(98.78 \%)$, PPV (99.16\%) and NPV (90.92\%) were observed with FPTB in comparison with LJ culture for both respiratory tract and non-respiratory tract specimens. In the current study, both LJ and MGIT recovered five nontuberculous mycobacteria from respiratory specimens $(n=145)$ and three nontuberculous mycobacteria from non-respiratory tract specimens $(n=44)$, but these samples were negative in the FPTB assay and AFB smear. Several possibilities exist to explain this result; phage attachment and replication in the FPTB assay depends on the number of viable bacilli and intact phage receptors on the cell surface and an effective phage attachment and replication depend on the strain of TB or the physiological state of the bacilli (Albert et al. 2002).

As shown in Table II, a total of 117 cases were identified by the FPTB assay from all mycobacterial isolates recovered from respiratory tract specimens $(n=145)$. The presence of disease was determined by clinical, radiological and other laboratory findings suggestive of TB. When comparing clinically evident respiratory tract cases $(n=208)$ with different diagnostic techniques, the percentage of organisms detected was found to be $52.4 \%$, $61.06 \%, 56.25 \%$ and $63.46 \%$ in AFB, LJ, FPTB assays and MGIT, respectively. The level of detection in the FPTB assay was found to be relatively low (56.25\%) in comparison to LJ and MGIT. This is most likely due to the paucibacillary nature of the specimen. Concurrently, among the non-respiratory specimens processed ( $\mathrm{n}=$ $119)$, the detection rate was found to be $15.13 \%, 32.77 \%$ and $35.29 \%$ in AFB, LJ and MGIT, respectively. However, in FPTB, the level of detection was found to be much lower $(26.89 \%)$. Moreover, it is reported that the analytical sensitivity of the phage assay is generally in the range of $100-300 \mathrm{bacilli} / \mathrm{mL}$ of specimen, whereas the culture of mycobacteria can detect 10-100 bacilli $/ \mathrm{mL}$ (Biotec

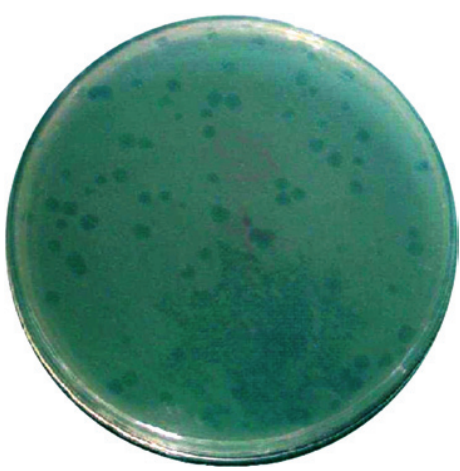

Photograph of positive plate from Biotec FASTPlaque TB ${ }^{\mathrm{TM}}$ test.

Laboratories 2000a, b). This suggests that the phage assay is not useful in direct detection of M. tuberculosis using paucibacillary specimens. However, studies by other investigators suggest that intensifying the original number of organisms present in the sample by culturing it for a few days in liquid media may boost the sensitivity in paucibacillary specimens (Shenai et al. 2002).

Interestingly, it has also been observed that none of the culture systems independently identified all the mycobacteria isolates from respiratory $(n=145)$ and non-respiratory tract specimens $(n=44)$. However, the maximum number of isolates was detected in MGIT and LJ culture (Table II). The above results indicate that the combination of these methods (broth and solid media) could be the most feasible approach to identify mycobacterial infection.

In summary, the FPTB assay was unable to detect nontuberculous mycobacteria and few M. tuberculosis complex cases from paucibacillary clinical specimens, even though this technique is simple, economical and less time consuming than traditional methods. The above results also signify that the analytical sensitivity of the FPTB assay is reasonably low and may not be suitable for the direct detection of TB using paucibacillary specimens. However, it was also observed that none of the systems recovered all isolates independently and the highest recovery rates were noted when solid medium was combined with liquid medium. The current study concludes that MGIT has been proven as a dependable, efficient system for recovery of both $M$. tuberculosis complexes and nontuberculous mycobacteria from pulmonary (sputum with scanty bacilli or clinically evident, smear negative cases) and paucibacillary extra-pulmonary TB when used in combination with LJ media.

\section{REFERENCES}

Albert H, Heydenrych A, Brookes R, Mole RJ, Harley B, Subotsky E, Henry R, Azevedo V 2002. Performance of a rapid phagebased test, FASTPlaque TB, to diagnose pulmonary tuberculosis from sputum specimens in South Africa. Int J Tuberc Lung Dis 6: 529-537.

Allen BW 1984. Tuberculosis bacteriology in developing countries. Med Lab Sci 41: 400-409.

Badak FZ, Kiska DL, Setterquist S, Hartley C, O'Connell MA, Hopfer RL 1996. Comparison of Mycobacteria Growth Indicator Tube 
with BACTEC 460 for detection and recovery of mycobacteria from clinical specimens. J Clin Microbiol 34: 2236-2239.

Biotec Laboratories 2000a. Biotec FASTPlaque TB TM 2000. A rapid bacteriophage assay for detection of Mycobacterium complex in clinical samples. Instruction Manual, Biotec, Ipswich, 15 pp.

Biotec Laboratories 2000b. Biotec FASTPlaque TB ${ }^{\mathrm{TM}}$ 2000. An innovative breakthrough for the detection of tuberculosis. Technical data report, Biotec, Ipswich, 9 pp.

Cheng VC, Yew WW, Yuen KY 2005. Molecular diagnostics in tuberculosis. Eur J Clin Microbiol Infect Dis 24: 711-720.

Dorman SE 2010. New diagnostic tests for tuberculosis: bench, bedside and beyond. Clin Infect Dis 3: 173-177.

Hanna BA, Ebrahimzadeh A, Elliott LB, Morgan MA, Novak SM, Rusch-Gerdes S, Acio M, Dunbar DF, Holmes TM, Rexer CH, Savthyakumar C, Vannier AM 1999. Multicenter evaluation of the BACTEC MGIT 960 system for recovery of mycobacteria. J Clin Microbiol 37: 748-752.

Hillemann D, Richter E, Rüsch-Gerdes S 2006. Use of the BACTEC Mycobacteria Growth Indicator Tube 960 automated system for recovery of mycobacteria from 9,558 extrapulmonary specimens, including urine samples. J Clin Microbiol 44: 4014-4017.

Kandi S, Madhavi G 2010. Emerging trends in the diagnosis of tuberculosis - RNTCP and beyond. J Indian Med Assoc 108: 368-370.

Kent PT, Kubica GP 1985. Public health mycobacteriology - a guide for the level III laboratory, Department of Health and Human Serv-
ices/Centers for Disease Control and Prevention, Atlanta, 207 pp.

Marei AM, El-Behedy EM, Mohtady HA, Afify AF 2003. Evaluation of a rapid bacteriophage-based method for the detection of Mycobacterium tuberculosis in clinical samples. J Med Microbiol 52: 331-335.

McNerney R 1996. Diagnosis: present difficulties and prospects for the future. Afr Health 19: 22-23.

Nancy DE, John B, Paula F, Philip H, Robert CH, Max S, Patricia MS 2000. Diagnostics standards and classification of tuberculosis in adults and children. Am J Respir Crit Care Med 161: 1376-1395.

Otive-Igbuzor EJ 2002. Phage amplification technology in the diagnosis of pulmonary tuberculosis: applicability in Nigeria. Afr $J$ Clin Exp Microbiol 3: 55-63.

Pfyffer GE, Welscher HM, Kissling P, Cieslak C, Casal MJ, Gutierrez J, Rüsch-Gerdes S 1997. Comparison of the Mycobacteria Growth Indicator Tube (MGIT) with radiometric and solid culture for recovery of acid-fast bacilli. J Clin Microbiol 35: 364-368.

Shenai S, Rodrigues C, Mehta AP 2002. Evaluation of new phage amplification technology for rapid diagnosis of tuberculosis. Indian J Med Microbiol 20: 194-199.

WHO - World Health Organization 2010. Tuberculosis. Available from: who.int/mediacentre/ factsheets/fs104/en/index.html.

Wilson SM, Suwaidi ZA, McNerney R, Porter J, Drobniewski F 1997. Evaluation of a new rapid bacteriophage-based method for the drug susceptibility testing of Mycobacterium tuberculosis. Nat Med 3: 465-468. 